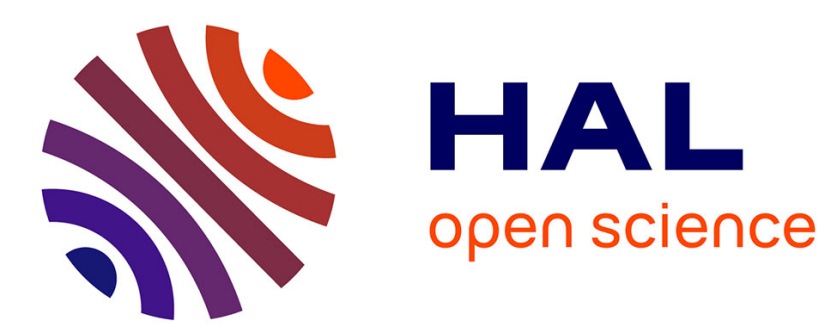

\title{
High-Tc superconductors as almost-localized systems: Resonance and fluctuations
}

M. Lavagna

\section{To cite this version:}

M. Lavagna. High-Tc superconductors as almost-localized systems: Resonance and fluctuations. Physica C: Superconductivity, 1988, 153-155, pp.1271 - 1272. 10.1016/0921-4534(88)90276-6 . hal01896299

\section{HAL Id: hal-01896299 \\ https://hal.science/hal-01896299}

Submitted on 17 Oct 2018

HAL is a multi-disciplinary open access archive for the deposit and dissemination of scientific research documents, whether they are published or not. The documents may come from teaching and research institutions in France or abroad, or from public or private research centers.
L'archive ouverte pluridisciplinaire HAL, est destinée au dépôt et à la diffusion de documents scientifiques de niveau recherche, publiés ou non, émanant des établissements d'enseignement et de recherche français ou étrangers, des laboratoires publics ou privés. 
${ }_{\text {HIGH-T }}$ SUPERCONDUCTORS AS ALMOST-LOCALIZED SYSTEMS : RESONANCE AND FLUCIUATIONS

Mireille LAv̄AGĩ̄a

Laboratoire Louis iNéel, C.N.R.S., 166 X, 38042 GRENOBLE-CEDEX, FRANCE.

A new approach is developed to treat the Mott localisation in strongly-correlated systems. It is based on a perturbation theory on the Hubbard model as a function of the superexchange coupling $\mathrm{J}$. The Hartree term which is proved to correspond to the Gutzwiller approximation constitutes the natural ground state around which the system fluctuates. It is shown how superconductivity arises from intersite correlations developed by quantum fluctuations around a re sonant state.

One of the consequences of the discovery of the new high-T $T_{c}$ superconductors has been to reactualize the old - but not solved - problem of magnetism and localization 1 (in the sense of Mott) in strongly-correlated systems. $\mathrm{La}_{2} \mathrm{CuO}_{4}$, and probably $\mathrm{YBa}_{2} \mathrm{Cu}_{3} \mathrm{O}_{6}$ - as suggested by recent neutron diffraction experiments - are antiferromagnet insulators, and superconductivity appears to be induced by doping. By the simplicity of its formulation, the Hubbard model is particularly attractive and it gave rise to a great deal of work these last months. Anderson 2 followed by others proposed to start from the completely localized limit large $U$ in the half-filled band case - giving rise to the so-called resonant-valence-bond (RVB) state. Further support 3 has been provided by performing a mean-field theory on the canonically transformed hamiltonian in the large U limit. All these theories clearly show the role of the superexchange in the origin of superconductivity. However, as stressed by their authors, they need further improvements to include quantum fluctuations and describe the dynamic of vacancies when away from half-filling.

We develop here a different point of view, and propose that the Gutzwiller approximation (G.A.) $1,4,5$ is a good starting point to describe the dynamic of vacancies. Besides its success, the G.A. presents some weaknesses (broken symmetry, absence of superexchange and of superconductivity) due to the variational nature of the method, which is shown to correspond to a saddle-point approximation 6 . Each of these failures should be corrected by adding quantum fluctuations. The aim of this paper is to propose an original method to take account of the quantum fluctuations around the ground state defined in the G.A. and to show how this is related to RVB.

Let us start from the formulation introduced by Kotliar-Ruckenstein 6 with the four operators $\alpha_{i}\left(e_{i}, p_{i \sigma}\right.$ and $d_{i}$ boson operators) on the empty, singly-occupied $\sigma$ and doubly-occupied i site.

$$
\begin{aligned}
& \begin{aligned}
H & =\sum_{i j \sigma} t_{i j} z_{i \sigma}^{+} z_{j \sigma} c_{i \sigma}^{+} c_{j \sigma}+U \sum_{i} d_{i}^{+} d_{i} \\
& +\sum_{i} \lambda_{i}^{(1)}\left(e_{i}^{+} e_{i}+\sum_{\sigma} p_{i \sigma}^{+} p_{i \sigma}+d_{i}^{+} d_{i}-1\right) \\
& +\sum_{i \sigma} \lambda_{i \sigma}^{(2)}\left(c_{i \sigma}^{+} c_{i \sigma}-p_{i \sigma}^{+} p_{i \sigma}-d_{i}^{+} d_{i}\right)
\end{aligned} \\
& \quad \text { with }: \\
& z_{i \sigma}=\left(1-e_{i}^{+} e_{i}^{-} p_{i-\sigma}^{+} p_{i-\sigma}\right)^{-y / z} \bar{z}_{i \sigma}\left(1-p_{i \sigma}^{+} p_{i \sigma}-d_{i}^{+} d_{i}\right)^{-1 / 2} \\
& \text { and } \bar{z}_{i \sigma}=e_{i}^{+} p_{i \sigma}+p_{i-\sigma}^{+} d i
\end{aligned}
$$

$\lambda_{i}^{(1)}$ and $\lambda_{i 0}^{(2)}$ are Lagrange multipliers to enforce the constraints. (1) is strictly equivalent to the Hubbard model with $z_{i o}$ specified by (2). It has then be shown that the saddle-point approximation 6 gives back the G.A.. Typically, there are two regimes (noting $\sum_{i j} t_{i j} c_{i \sigma j \sigma}^{+} c_{j \sigma}=\bar{\varepsilon}_{0} / 2=-U_{c} / 16$ in the paramagnetic half-filled band case) : $\begin{aligned} \text { Regime I : }(u & \left.=u / U_{c}<1 \text { and } n=1\right)^{4}: \\ d^{2} & =(1-u) / 4 \text { and } q=z^{2}=1-u^{2}\end{aligned}$

A Mott transition is expected for $U>U_{c}$ Regime II $\left(u=U / U_{c}>1\right.$ and $(1-n)=\delta\langle<1)^{5}$ :

$$
\begin{aligned}
& d^{2}=\delta\left(1-\zeta_{c}\right)^{2} / 4 \zeta_{c} \text { and } q=2 \delta / \zeta_{c} \\
& \text { with the notation } \zeta_{c}=\sqrt{1-1 / u}
\end{aligned}
$$

We emphasize that the resolution of the saddle-point equations can also lead to the determination of the Lagrange multipliers ( $\lambda_{\sigma}$ is also the chemical potential):

$$
\begin{aligned}
\text { Regime I : } \lambda^{(1)}=U_{c}(1+u)(2-u) / 4 \text { and } \lambda_{\sigma}^{(2)}=U / 2 \\
\text { Regime II : } \left.\lambda^{(1)}=\lambda_{\sigma}^{(2)}=U(1 \pm \sqrt{1-1 / u}) / 2\right) \\
\text { (+ and }- \text { for } n>1 \text { and } n<1 \text { respectively) }
\end{aligned}
$$


The immediate consequence is the formation of a gap (Mott gap) for $u>1$ of width $U \sqrt{1-1 / u}$. By analogy with the Abrikosov-Suhl resonance of the Kondo problem, we interpret, the renormalisation of the band width to $2 \mathrm{Wq}$ as a resonance pinned at the Fermi level, and cut by a gap for $u>1$. Both resonances are typical of almost-localized systems.

The different hopping terms of (1) (in $e^{2} p^{2}, p^{2} d^{2}$ and $\left.e p^{2} d\right)$ bind sites of occupations both $n \leq 1$, both $n \geq 1$ and $n \leq 1, n \geq 1$ respectively. In the radial gauge ( $\lambda^{(2)}$ becoming an operator), we demonstrate that the saddle-point approximation is equivalent to a mean-field theory where bosons are treated one at each time as a propagator :

$t D^{-1} e p^{2} \alpha=\sum_{\alpha, \beta=e, p_{\sigma}, d} \frac{J_{\alpha \beta} D^{-2}}{t} \frac{\left(e p^{2} d\right)^{2}}{\alpha \beta}\left|\bar{\varepsilon}_{0}\right|(4)$

where $D=\bar{z}_{i \sigma} / z_{i \sigma}$ and $J_{\alpha \beta}=t^{2} / D_{\alpha \beta}^{0}$

We introduce in this way a generalized superexchange coupling :

$J^{*}=\sum_{\alpha \beta} J_{\alpha \beta} D^{-2} \frac{\left(e p^{2} d\right)^{2}}{\alpha \beta}=\frac{t^{2}}{\left|\overline{\varepsilon_{0}}\right|} \frac{e p^{2} d}{(e p+p d)^{2}} q$

Besides the $e^{2} p^{2}$ and $p^{2} d^{2}$ terms which can be treated in mean-field, the effective hamiltonian contains an intersite interaction term and can be written :

$H_{e f f}=\sum_{i j \sigma}\left(e^{2} p_{\sigma}^{2}+p_{-\sigma}^{2} d^{2}\right) D^{-1} c_{i \sigma}^{+} c_{j \sigma}$

$+J^{*}\left(4 \vec{S}_{i} \cdot \vec{s}_{j}-n_{i} \cdot n_{j}\right)+$ (Lagrangian terms)

We have just demonstrated that the Hartree treatment of the intersite term gives back the saddle-point $e p^{2} d$ term, and ${ }_{*}$ so the Gutzwiller approximation. Note that $J=\left(2 t^{2} / U\right) q$ in regime II leading for $U=\infty$ to the usual canonically transformed Hubbard hamiltonian (RVB state for $n=1$ ). The interest of the formulation in eq. 6 goes beyong the G.A. and the intersite interaction can be attacked by the standard methods of the many-body problem. Due to the short-ranged nature of the potential (intersite correlations), the essential contributions are to be found in the ladder approximation, in contrast to the ring approximation used in the $1 / \mathrm{N}$ Anderson model 7 . This provides an interaction between particles of opposite spins whose decomposition in cubic harmonics is estimated to :

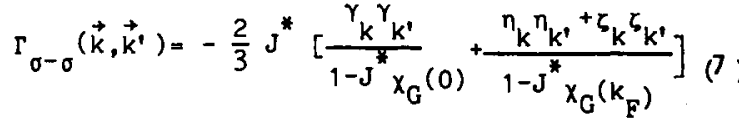

where $\gamma_{k}$ and $\eta_{k}, \zeta_{k}$ have respectively the $s-$ and d-wave symmetry with a maximal weight at $k=0$ and $k=k$ respectively. This leads to an

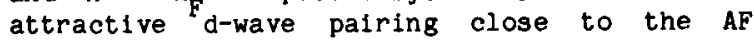
instability. High $\mathrm{T}_{\mathrm{c}}$ are explained by the large value of the cut-off provided by the renormalized bandwidth qW.However, a compromise has to be found since the superconducting pairing $\rho_{0} q^{-1} \Gamma=\rho_{0}\left(2 t^{2} / U\right) /(1+\delta)$ [for $u>1$ and $\delta$ $<$ 1] decreases with $\delta$, oppositely to the cut-off. These competitive effects lead to a maximum of $\mathrm{T}_{c}$ with $\delta$ as in ref. (3) but with a more natural starting point $T_{c}=0$ for $\delta=0$ due to the effects of localisation at half-filling $(q=0)$.

To conclude, we have shown how a perturbation theory can be developed for the Hubbard model at any value of the coupling, the relevant interaction term coming from the super-exchange $\mathrm{J}^{*}$. The identification of the Gutzwiller approximation with the Hartree term constitutes a useful result which permits to find back any feature of the G.A. (metamagnetism, magnetization curve, thermodynamics ...). This constitutes the natural ground state in which describe the dynamic of vacancies. The appearance of a resonance pinned at the Fermi level clearly shows the analogy with another class of almost-locallzed systems i.e. the heavy fermions. In both cases, superconductivity may arise from intersite correlations developed by quantum fluctuations around a resonant state.

Acknowledgements : I wish to express my gratitude to Prof. P. Nozieres for valuable discussions and encouragement.

\section{REFERENCES}

(1) D. Vollhardt, Rev.Mod.Phys. 56 (1984) 99.

(2) P.W. Anderson, Sclence 235 (1987) 1196

(3) G. Baskaran, Z. Zou, P.W. Anderson, Sol.St.Comm. 63 (1987) 973 A.E. Ruckenstein, P.J. Hirschfeld, J. Appel Phys.Rev.B 36 (1987) 857

(4) M.C. Gutzwiller, P.R.L. 10 (1963) 159 W.F. Brinkman, T.M. Rice, $\overline{\text { P.R.B. }} 2$ (1970) 4302

(5) D. Vollhardt, P. Wolfe, P.W. Añderson P.R.B., 35 (1987) 6703

(6) G. Kotli lar, A.E. Ruckenstein, P.R.L. 57 (1986) 1362

(7) M. Lavagna, A.J. Millis, P.A. Lee P.R.L. $\underline{58}$ (1987) 266 . 